\title{
Mass ratio in SS433 revisited
}

\author{
A.M. Cherepashchuk ${ }^{1 \star}$, K.A. Postnov ${ }^{1,2} \dagger$ and A.A. Belinski ${ }^{1} \ddagger$ \\ 1 Sternberg Astronomical Institute, M.V. Lomonosov Moscow State University, 13, Universitetskij pr., 119234, Moscow, Russia \\ 2 Kazan Federal University, Kremlevskaya 18, 420008 Kazan, Russia
}

Accepted XXX. Received YYY; in original form ZZZ

\section{INTRODUCTION}

Long-term optical, X-ray and radio studies of the microquasar SS433 have suggested that this unique Galactic object is a high-mass eclipsing X-ray binary at advanced evolutionary stage (Margon et al. 1979; Cherepashchuk 1981; Fabrika 2004). In SS433, a supercritical accretion disc and relativistic jets precessing with a period of $P_{\text {prec }} \simeq 162^{\mathrm{d}} .3$ are observed. A recent analysis of the 40-years optical data on Doppler shifts of moving $H_{\alpha}$ emission lines (Cherepashchuk et al. 2018a) revealed that the precessional, nutational and orbital periods of SS433 are on average stable over 40 years. The SS433 binary system is completing the secondary mass transfer stage after which a WR+C binary should be formed (C here means the relativistic compact object). However, at odds with predictions of the standard theory of evolution of massive close binaries (Massevich \& Tututkov 1988), no common envelope has been formed in the system, and the excess of mass, energy and angular momentum is carried away from the binary system by a powerful stellar wind $\left(\dot{M} \approx 10^{-4} M_{\odot} \mathrm{yr}^{-1}, v \simeq 1500 \mathrm{~km} \mathrm{~s}^{-1}\right)$ launched from the precessing accretion disc, as well as by relativistic jets $\left(\dot{M} \approx 10^{-6}-10^{-7} M_{\odot} \mathrm{yr}^{-1}, v \simeq 0.26 c\right)$.

Recently, important details of the high-mass close binary evolution have been recognized enabling us to better understand this unique feature of SS433 (van den Heuvel et al. 2017; Pavlovskii \& Ivanova 2015; Pavlovskii et al. 2017). According to van den Heuvel et al. (2017), the common envelope in a massive X-ray binary can form if the component mass ratio $q=M_{\mathrm{x}} / M_{\mathrm{V}} \lesssim 0.29\left(M_{\mathrm{X}}\right.$ and $M_{\mathrm{V}}$ is the

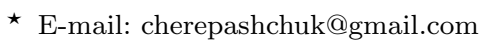

$\dagger$ E-mail: pk@sai.msu.ru

$\doteqdot$ E-mail: aleks@sai.msu.ru
}

mass of the relativistic and optical component, respectively). In the opposite case, $q \gtrsim 0.29$, the common envelope is not formed and the binary system evolves as a semi-detached one with a steady mass transfer from the Roche-lobe filling optical star forming a supercritical accretion disc with powerful stellar wind outflow, like in SS433.

The stability of mass transfer through the vicinity of the inner Lagrangian point $\mathrm{L}_{1}$ was investigated in several papers (see, for example, Pavlovskii \& Ivanova (2015); Pavlovskii et al. (2017)). It was shown that for massive donors with radiative envelopes in high-mass X-ray binaries and provided that $M_{\mathrm{X}} \gtrsim 0.29 M_{\mathrm{V}}$, the mass transfer through $\mathrm{L}_{1}$ is stable. In this case, the optical star can remain for a long time in the state of significant overfilling of its Roche lobe, and the matter from the star can be lost through both inner $L_{1}$ and outer $\mathrm{L}_{2}$ Lagrangian points. The effective photosphere radius of the optical star can significantly exceed that of its mean Roche lobe. In the case of SS433, this enables an anomalously large width of the X-ray (2-10 keV) eclipse of relativistic jets (Kawai et al. 1989; Kotani et al. 1996) to be explained with a relatively large binary mass ratio $q$. Thus, determination of the binary mass ratio in SS433 is very important to understand the evolution of this unique Galactic system.

A surprising feature of SS433 is the stability of the orbital binary period (see Fabrika (2004), Cherepashchuk et al. (2018b) (Paper I)), which suggests a high binary mass ratio $q \gtrsim 0.6$. Recent spectrophotometric and astrometric observations of SS433 by the GRAVITY VLT interferometer with an angular resolution of better than one milliarcsecond (Waisberg et al. 2018) enabled sky mapping of the region of the double-peak Brackett $\mathrm{Br}-\gamma$ emission line in the IRspectrum of the system. These direct observations proved, for the first time, that these emission lines are not gener- 
ated in the accretion disc around the compact object but are produced in the circumbinary disc-like shell around the binary system. It is also proved that the broad component of these double-peak emission lines in SS433 is produced by the stellar wind outflow from the supercritical accretion disc. Therefore, the conclusion of our Paper I, in which a complex model of the formation of Brackett emission line profiles was suggested (double-peak component - in the circumbinary shell, broad lines - in the supercritical accretion disc wind) is now supported by direct observations.

According to Waisberg et al. (2018), the circumbinary shell around SS433 demonstrates a super-Keplerian motion with a significant radial velocity component, apparently suggesting a replenishment of the rotating shell by matter from the supercritical accretion disc wind outflow with a velocity of $\sim 1000 \mathrm{~km} \mathrm{~s}^{-1}$.

In Paper I, we estimated the binary mass ratio in SS433 $q \gtrsim 0.6$ from the observed constancy of the binary orbital period using the model of isotropic mass re-emission for the supecritical accretion disc wind and assuming an additional mass loss from the system through the outer Lagrangian point $\mathrm{L}_{2}$. As the geometry and structure of Brackett emission line region is now established from the GRAVITY VLTI observations (Waisberg et al. 2018), we are able to obtain a more reliable binary mass ratio estimate in SS433 from the observed constancy of the binary orbital period: $q \gtrsim 0.6$. This new estimate is consistent with conclusions of van den Heuvel et al. (2017) on the absence of common envelope in SS433 and confirms the status of SS433 as a semi-detached high-mass X-ray binary with stable mass transfer onto a massive compact object. Note that a high mass of the compact object in SS433 was also independently obtained in the recent paper by Bowler (2018).

When estimating the mass of the relativistic object in SS433 one should bear in mind that the distance to the system is well known, $d=5.5 \mathrm{kpc}$ (Blundell \& Bowler 2004), and the total absorption in the direction to SS433 in the optical is $A_{\mathrm{v}} \simeq 8^{\mathrm{m}}$ (Cherepashchuk et al. 1982; Margon 1984). Using this information, from the visible stellar magnitude of SS433 and by fixing the fraction of the optical emission from the supercritical accretion disc $(\sim 0.6-0.7$, Hillwig \& Gies (2008)), it is possible to estimate the mass of the optical component, which, according to Goranskij (2011), falls within the range $M_{\mathrm{V}}=8.3-12.5 M_{\odot}$, corresponding to the spectral class A4I-A8I. An upper mass limit of the optical component in SS433 can be set at $M_{\mathrm{V}}=15 M_{\odot}$. Therefore, the binary mass ratio $q>0.6$ implies the mass of the relativistic compact star to be $M_{\mathrm{x}}=q M_{\mathrm{V}}>(5-9) M_{\odot}$, i.e. the compact star in SS433 must be a black hole.

\section{A NEW ESTIMATE OF THE BINARY MASS RATIO IN SS433}

Like in Paper I, consider the following model of mass flows in SS433. We will assume a circular binary with orbital separation $a$. Let the mass-loss rate from the optical star $M_{\mathrm{v}}$ be $\dot{M}_{\mathrm{v}}$, the total mass of the system be $M=M_{\mathrm{V}}+M_{\mathrm{X}}=M_{\mathrm{V}}(1+q)$, the mass accreted by the compact object be 0 , the mass outflow from the supercritical accretion disc via the Jeans mode (i.e. with the specific orbital angular momentum of the accretor) be $\beta \dot{M}_{\mathrm{v}}, \beta \leq 1$, and the mass outflow from the system in a

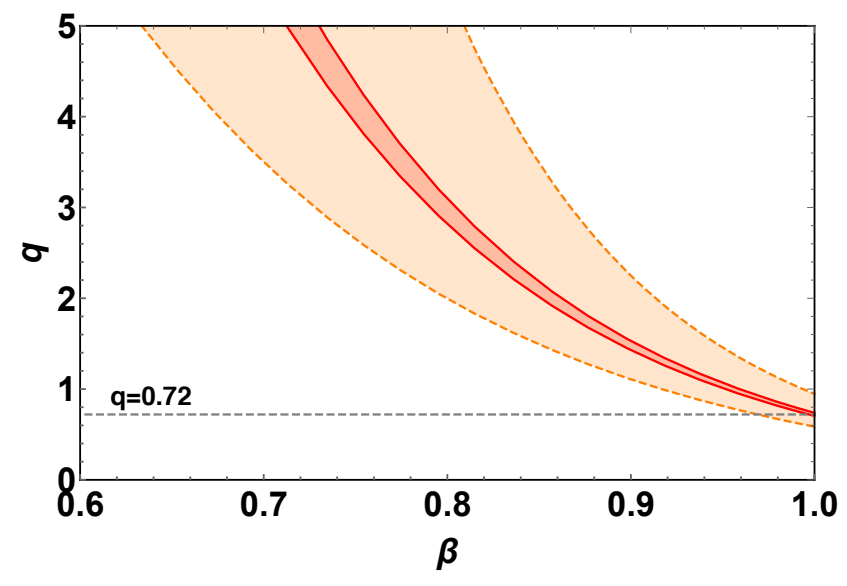

Figure 1. Constraints on the mass ratio of SS433 for different values of the mass-loss rate $\dot{M}_{\mathrm{v}}=10^{-4}$ and $10^{-5} M_{\odot} \mathrm{yr}^{-1}$ (intervals restricted by the solid and dashed lines, respectively), for the fiducial value of the dimensionless parameter $K=5$. The dashed horizontal line shows the solution of the quadratic equation $3 q^{2}+$ $2 q-1=0$ corresponding to $\dot{P}_{\mathrm{b}}=0$ at $\beta=1$ (only Jeans mode mass outflow) independently of the mass-loss rate.

circumbinary shell be $(1-\beta) \dot{M}_{\mathrm{v}}$. Then from the angular momentum conservation we obtain the equation for the change of the binary separation (see Paper I):

$\frac{\dot{a}}{a}=-2\left(1-\frac{1}{2} \frac{M_{\mathrm{V}}}{M}\right) \frac{\dot{M}_{\mathrm{v}}}{M_{\mathrm{v}}}+2 \beta \frac{M_{\mathrm{V}}}{M_{\mathrm{x}}} \frac{\dot{M}_{\mathrm{v}}}{M}+2(1-\beta) \frac{\left(\frac{d J}{d t}\right)_{\text {out }},}{J}$,

where $J=\left(M_{\mathrm{X}} M_{\mathrm{V}} / M\right) \sqrt{G M a}$ is the orbital angular momentum of the binary. Conservative mass transfer corresponds to $\beta=0$ and $d J=0$, and in the non-conservative case $(d J / d t)_{\text {out }} / J$ specifies the additional orbital angular momentum loss by the mass escaping the binary in the outer shell.

In Paper I, we have assumed a model for the term $(d J / d t)_{\text {out }} / J$ with mass-loss carrying the specific angular momentum corresponding to that at the outer Lagrangian point $\mathrm{L}_{2}$ (see, e.g., Soberman et al. (1997)). New measurements (Waisberg et al. 2018) enable us to specify this term directly from observations. Indeed, this term can be represented in the form

$\left(\frac{d J}{d t}\right)_{\text {out }}=(1-\beta) \dot{M}_{\mathrm{v}} v_{\phi}\left(R_{\text {out }}\right) R_{\text {out }}$,

where $v_{\phi}\left(R_{\text {out }}\right)$ is the tangential component of the outflow velocity at the outer radius of the circumbinary shell $R_{\text {out }}$. Therefore, the last term in Eq. (1) takes the form:

$2(1-\beta) \frac{\left(\frac{d J}{d t}\right)_{\text {out }}}{J}=2 \frac{\dot{M}_{\mathrm{v}}}{M_{\mathrm{v}}} \frac{M}{M_{\mathrm{X}}} \frac{(1-\beta) v_{\phi}\left(R_{\mathrm{out}}\right) R_{\mathrm{out}} \omega^{1 / 3}}{(G M)^{2 / 3}}$,

where $\omega=2 \pi / P_{\mathrm{b}}$ is the orbital angular frequency and we have used the 3rd Kepler's law to express the orbital separation through $\omega$.

Changing from $\dot{a} / a$ to $\dot{P}_{b} / P_{b}$ with the help of the 3 rd Kepler's law and substituting $M_{\mathrm{X}}=q M_{\mathrm{V}}, M=M_{\mathrm{V}}(1+q)$, we ultimately arrive at the equation for the fractional change of the binary orbital period (cf. Eq.(7) in Paper I):

$\frac{\dot{P}_{b}}{P_{b}}=-\frac{\dot{M}_{\mathrm{v}}}{M_{\mathrm{v}}} \frac{3 q^{2}+2 q-3 \beta-3 K(1-\beta)(1+q)^{5 / 3}}{q(1+q)}$. 


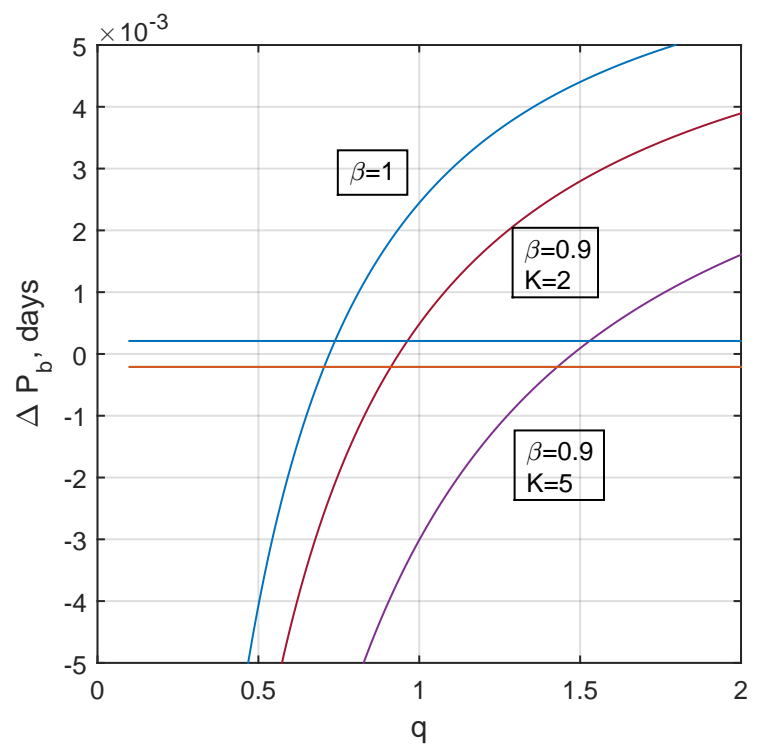

Figure 2. Expected change in the orbital period of SS433 (in days) as a function of the mass ratio $q$ (Eq. (4)) over a 30-yr period. Shown is the limiting case of purely Jeans mode outflow $(\beta=$ $1)$ and the case of additional $10 \%$ mass-loss $(\beta=0.9)$ via the circumbinary shell parametrized by the parameter $K=5$ and $K=2$ (Eq. (6)) for the assumed total mass-loss rate $\dot{M}_{\mathrm{V}}=10^{-4} M_{\odot} \mathrm{yr}^{-1}$. Two parallel lines show the boundaries $\Delta \boldsymbol{P}_{\mathrm{b}}= \pm 3 \sigma_{P}=0^{\mathrm{d}} .00021$ derived from observations.

Here the dimensionless coefficient $K$ specifying the angular momentum loss via circumbinary disc reads

$K=\frac{v_{\phi}\left(R_{\text {out }}\right) R_{\text {out }}}{\left(G M_{\mathrm{V}}\right)^{2 / 3}}\left(\frac{2 \pi}{P_{b}}\right)^{1 / 3}$.

Taking the orbital period $P_{b}=13^{\mathrm{d}} .1$ and normalizing $v_{\phi}$ and $R_{\text {out }}$ to the characteristic values $220 \mathrm{~km} \mathrm{~s}^{-1}$ and 0.7 mas (see Table 3 in Waisberg et al. (2018), the 'outflow' model favoured by the authors), we obtain

$K \approx 5.1\left(\frac{v_{\phi}\left(R_{\text {out }}\right)}{220 \mathrm{~km} \mathrm{~s}^{-1}}\right)\left(\frac{R_{\text {out }}}{0.7 \mathrm{mas}}\right)\left(\frac{M_{\mathrm{v}}}{15 M_{\odot}}\right)^{-2 / 3}$.

The large value of $K$ (cf. $x \simeq 1$ in Eq. (7) of Paper 1) is very unusual. Indeed, taking at face value, this suggests a strong increase of the specific angular momentum in the outflow compared to the orbital value. The physical mechanism for that, as the authors Waisberg et al. (2018) stress, remains unknown. Moreover, the circumbinary outflow in SS433 is observed to be highly variable. Nevertheless, in our analysis below we use $K=5$ as a fiducial value.

Acting exactly in the same way as in Paper I, from the observed stability of the orbital period of SS433 over $\Delta t \sim 28$ years, $\left|\dot{P}_{b}\right| \leq 3 \times \sigma_{P} / \Delta t$, where $\sigma_{P}=0^{\mathrm{d}} .00007$ is the uncertainty in the SS433 orbital period determination (Goranskij 2011), and assuming the optical star mass $M_{\mathrm{V}}=$ $15 M_{\odot}$ with the mass-loss rate $\dot{M}_{\mathrm{v}}=10^{-4} M_{\odot} \mathrm{yr}^{-1}$, in Figure 1 we plot the constraints on the binary mass ratio in SS433 $q$ for $K=5$, as a function of the parameter $\beta$.

Clearly, even a tiny fraction of the total mass loss via the circumbinary shell, $1-\beta \simeq 0.01$, places a mass ratio constraint $q \gtrsim 0.6$ in SS433. In view of high sensitivity of

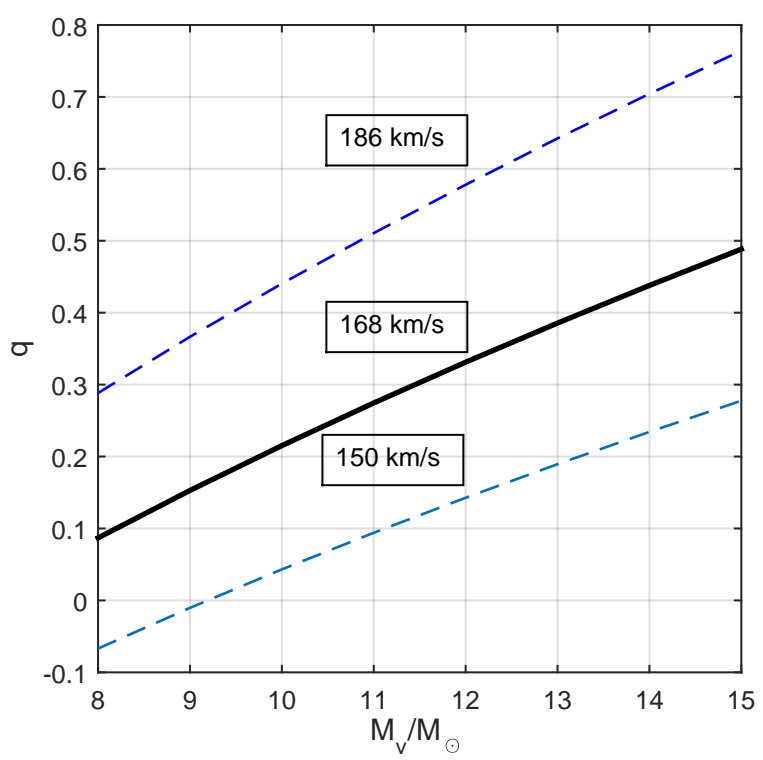

Figure 3. Binary mass ratio $q$ corresponding to the radial orbital velocity of the compact object (Eq. (7)) as required to reproduce observed HeII velocity amplitude $V_{\text {HeII }}=168 \pm 18 \mathrm{~km} \mathrm{~s}^{-1}$.

this estimate to the amount of matter outflow through the circumbinary shell, it is important to independently evaluate $(1-\beta) \dot{M}_{\mathrm{v}}$. For example, the estimate of the disc-like mass outflow rate from radio observations (Blundell et al. 2001) suggests $\dot{M}_{\text {out }} \sim 0.18 \times 10^{-4} M_{\odot} \mathrm{yr}^{-1}$ (see their Eq. 1) for the source distance $d=5.5 \mathrm{kpc}$ and the outflow radial velocity at the radio optically thick distance $\sim 10^{15} \mathrm{~cm}$ of about $\sim 230 \times(3.5 / 60) \simeq 14 \mathrm{~km} \mathrm{~s}^{-1}$ (assuming the law $1 / r$ for the outflow velocity as in Waisberg et al. (2018)). Therefore, a few percents of the total mass loss rate from the system are quite possibly being ejected via the circumbinary shell outflow.

In Figure 2 we plot the expected change in the orbital period of SS433 (in days) over a 30-yr time interval as a function of the mass ratio $q$ calculated directly by formula eq. (4) for the limiting case of purely Jeans mode outflow $(\beta=1)$ and the case of additional $10 \%$ mass-loss $(\beta=0.9)$ via the circumbinary shell parametrized by the angular momentum loss parameter $K=5$ and $K=2$ (see Eq. (6)). Two parallel lines show the boundaries $\Delta P_{\mathrm{b}}= \pm 3 \sigma_{P}=0^{\mathrm{d}} .00021$. Clearly, in all cases the binary mass ratio cannot be smaller than $q_{\min } \sim 0.6$.

\section{DISCUSSION AND CONCLUSION}

In the present paper, we have re-analyzed the mass ratio constraints in SS433 imposed by the observed stability of the binary orbital period $P_{\mathrm{b}}$ over $\sim 30 \mathrm{yr}$ period of observations (Goranskij 2011) with taking into account the new GRAVITY VLTI result on the observed mass-loss through the circumbinary shell in this system (Waisberg et al. 2018). This analysis strengthens our previous conclusion (see Paper I) that the binary mass ratio $q=M_{\mathrm{x}} / M_{\mathrm{V}}$ in SS433 is likely to be $q \gtrsim 0.6$. The result is very sensitive to the (unknown) 
fraction of the mass-loss from the system via the circumbinary shell, the factor $(1-\beta)$ in Eq. (4). Extant measurements carried out at different epochs suggest strong variability of both the mass-loss rate and geometry of the outflow (Gravity Collaboration et al. 2017; Waisberg et al. 2018). In spite of these uncertainties, even with a tiny or zero average massloss rate via circumbinary disc, the observed orbital period stability over about 30 years strongly points to a high binary mass ratio. It is also important to stress that irrespective of details, the angular momentum transfer from the binary system through a circumbinary shell or a mass-loss through $\mathrm{L}_{2}$ point (or both) only increase the mass ratio estimate.

An independent constraint on the binary mass ratio can be obtained from the observed radial velocity amplitude of HeII emission lines. The radial orbital velocity of the compact object in SS433 for the mean binary inclination $i=79^{\circ}$ (Cherepashchuk et al. 2018a) reads

$V_{\mathrm{X}} \sin i \approx 219\left[\mathrm{~km} \mathrm{~s}^{-1}\right]\left(\frac{M_{\mathrm{V}}}{15 M_{\odot}}\right)^{1 / 3}(1+q)^{-2 / 3}$.

The observed radial velocity variations as measured by HeII emission lines, $V_{\mathrm{HeII}}=168 \pm 18 \mathrm{~km} \mathrm{~s}^{-1}$ (Hillwig et al. 2004), if interpreted as being purely due to the orbital motion of the compact object, requires the binary mass ratio $q$ as shown in Fig. 3. Clearly, within the measurement errors, the values $q \gtrsim 0.6$ inferred above can be accommodated for the optical component mass $M_{\mathrm{V}} \gtrsim 12 M_{\odot}$. For smaller masses, a controversy with the apparent lack of change of the binary orbital period would arise, even for insignificant mass loss through the circumbinary disk $(1-\beta \simeq 0)$. However, one should be careful in using HeII emission lines as a proxy for the orbital motion of the compact object because of their complicated formation in the inhomogeneous wind outflow (see, e.g., discussion and references in Robinson et al. (2017)).

The mass of the optical component in SS433 remains also debatable. Indeed, the interstellar absorption in the direction to SS433, $A_{\mathrm{V}} \approx 8^{m}$, is very large and is estimated with significant errors, and the contribution of the accretion disc to the total optical luminosity of SS433 is also derived spectrophotometrically with significant uncertainty. Thus the mass estimate of the optical component from its bolometric luminosity is not very reliable. In addition, as noted by Bowler (2010, 2011), the absorption lines in SS433 spectrum can arise in the circumstellar shell and not in the optical star atmosphere, therefore the spectral classification and mass of the optical star can be questionable.

We also note that the derived lower limit on the mass ratio in SS433 $q \gtrsim 0.6$ pointing to a higher mass of the optical star (see Fig. 3) contradicts to the optical star's radial velocity curve based on its classification as an A supergiant (Hillwig et al. 2004; Kubota et al. 2010) and suggesting $q \lesssim 0.3$. Clearly, here more studies are required.

We stress that the observed highly unstable character of the outflow parameters in SS433 (see e.g. Blundell et al. (2011)) does not allow us to infer a more accurate estimate. Further observations can be helpful to put more precise limits on the binary mass ratio in this system. Nevertheless, even at the present stage of knowledge of the system parameters, the obtained binary mass ratio lower limit $q \gtrsim 0.6$ seems to be quite robust, placing the compact object in SS433 in the realm of Galactic stellar-mass black holes.

\section{ACKNOWLEDGEMENTS}

We thank the anonymous referee for useful notes and suggestions. The work of AMCh is supported by the RSF grant 17-12-01241 (analysis of constraints from X-ray eclipses and circumbinary shell). The work of KAP and AAB (derivation of the constraints on $q$ from observations, Section 2) is supported by the Program of development of M.V. Lomonosov Moscow State University (Leading Scientific School 'Physics of stars, relativistic objects and galaxies').

\section{REFERENCES}

Blundell K. M., Bowler M. G., 2004, ApJL, 616, L159

Blundell K. M., Mioduszewski A. J., Muxlow T. W. B., Podsiadlowski P., Rupen M. P., 2001, ApJL, 562, L79

Blundell K. M., Schmidtobreick L., Trushkin S., 2011, MNRAS, 417,2401

Bowler M. G., 2010, A\&A, 521, A81

Bowler M. G., 2011, A\&A, 531, A107

Bowler M. G., 2018, A\&A, 619, L4

Cherepashchuk A. M., 1981, MNRAS, 194, 761

Cherepashchuk A. M., Aslanov A. A., Kornilov V. G., 1982, Sov. Astron., 26, 697

Cherepashchuk A. M., Esipov V. F., Dodin A. V., Davydov V. V., Belinskii A. A., 2018a, Astronomy Reports, 62, 747

Cherepashchuk A. M., Postnov K. A., Belinski A. A., (Paper I) 2018b, MNRAS, 479, 4844

Fabrika S., 2004, Astrophysics and Space Physics Reviews, 12, 1

Goranskij V., 2011, Peremennye Zvezdy, 31

Gravity Collaboration et al., 2017, A\&A, 602, L11

Hillwig T. C., Gies D. R., 2008, ApJL, 676, L37

Hillwig T. C., Gies D. R., Huang W., McSwain M. V., Stark M. A., van der Meer A., Kaper L., 2004, ApJ, 615, 422

Kawai N., Matsuoka M., Pan H.-C., Stewart G. C., 1989, PASJ, 41,491

Kotani T., Kawai N., Matsuoka M., Brinkmann W., 1996, PASJ, 48,619

Kubota K., Ueda Y., Fabrika S., Medvedev A., Barsukova E. A., Sholukhova O., Goranskij V. P., 2010, ApJ, 709, 1374

Margon B., 1984, ARAA, 22, 507

Margon B., Ford H. C., Grandi S. A., Stone R. P. S., 1979, ApJL, 233, L63

Massevich A. G., Tututkov A. V., 1988, Evolution of Stars: Theory and Observations. Moscow: Nauka

Pavlovskii K., Ivanova N., 2015, MNRAS, 449, 4415

Pavlovskii K., Ivanova N., Belczynski K., Van K. X., 2017, MNRAS, 465, 2092

Robinson E. L., Froning C. S., Jaffe D. T., Kaplan K. F., Kim H., Mace G. N., Sokal K. R., Lee J.-J., 2017, ApJ, 841, 79

Soberman G. E., Phinney E. S., van den Heuvel E. P. J., 1997, A\&A, 327, 620

Waisberg I., Dexter J., Olivier-Petrucci P., Dubus G., Perraut K., 2018, arXiv e-prints,

van den Heuvel E. P. J., Portegies Zwart S. F., de Mink S. E., 2017, MNRAS, 471, 4256

This paper has been typeset from a $\mathrm{T}_{\mathrm{EX}} / \mathrm{LAT} \mathrm{TX}$ file prepared by the author. 